\title{
Cytokine Release Syndrome, CTCAE
}

National Cancer Institute

\section{Source}

National Cancer Institute. Cytokine Release Syndrome, CT CAE. NCI Thesaurus. Code C143394.

A disorder characterized by fever, tachypnea, headache, tachycardia, hypotension, rash, and/or hypoxia caused by the release of cytokines. 